\title{
METRIC CHARACTERIZATION OF ELLIPTIC SPACE
}

\author{
BY
}

LEONARD M. BLUMENTHAL

1. Introduction. A given space $R$ is characterized metrically among a given class $\{S\}$ of spaces when necessary and sufficient conditions, expressed wholly (and explicitly) in terms of the metric of an arbitrary space $S$ of $\{S\}$, are found which insure the existence of a distance-preserving mapping of $S$ onto $R$. The metric characterizations of euclidean and Hilbert spaces among the class of semimetric spaces (that is, the class of all spaces in each of which a non-negative real number $p q$ is attached as "distance" to every pair of "points" $p, q$ of the space in such a way that $p q=q p$, while $p q=0$ if and only if $p=q$ ) were given first by Karl Menger [5, pp. 113-141] $\left({ }^{1}\right)$ and later by W. A. Wilson [6], while hyperbolic and spherical spaces (and subsets) were metrically characterized by the writer $[1,2,3$ (chap. 3 )].

This paper presents metric characterizations for elliptic spaces of finite and infinite dimensions, and thus rounds out the metric study of euclidean and non-euclidean spaces by the addition of those spaces (that is, the elliptic) whose metric characterizations, in the sense defined above, have not previously been obtained.

The principal results of the paper are the Characterization Theorems IIV of $\S 6$. In laying the groundwork for the proofs of those theorems $(\$ \S 4,5)$ only those consequences of our assumptions ( $\$ 3$ ) which are necessary to that end are developed. The reader might find it interesting to derive, in the manner of this paper, further properties of elliptic space (for example, the existence of Clifford parallels) directly from our postulates, in the light of which some of the unusual features of the space (for example, the presence of congruent sets which are not superposable) are easily discerned. A great deal of elliptic geometry is capable of being developed from these axioms with almost trivial effort.

Our results are obtained by direct, elementary geometric arguments. Only the simplest properties of elliptic space are made use of, and no reference at all is made to topological theorems. The deepest metric-theoretic result used is the very well known theorem that a complete, convex, metric space is segmentally connected. It is hoped that the intention to make the paper substantially complete in itself has been realized.

2. Some properties of elliptic space. If we denote by $S_{n, r}$ the metrically

Presented to the Society, November 25, 1945; received by the editors August 2, 1945.

(1) Numbers within brackets refer to the Bibliography concluding the paper. 
convex (that is, geodesically metrized)( $\left.{ }^{2}\right) n$-dimensional boundary of the sphere of radius $r$ in euclidean space of $n+1$ dimensions, a model of the elliptic $n$-space $E_{n, r}$ with space constant $r$, useful for our purpose, is obtained by identifying diametrically opposite points of $S_{n, r}$.

Though, as the model suggests, elliptic and spherical spaces are closely related (small neighborhoods of $S_{n, r}$ being, in fact, congruently contained in $\left.E_{n, r}\right)$ the spaces differ in so many important respects (both topological and metric) when considered globally that the procedure which was effective for the metric characterization of the $S_{n, r}$ could not be expected to be applicable to elliptic space without essential modifications. It turns out that in order to characterize metrically those semimetric spaces which are congruently imbeddable in $E_{n, r}$ (the so-called subset problem) the methods used heretofore are wholly unsuitable, while for the characterization of the whole $E_{n, r}$ (the space problem) they must undergo significant alteration $\left({ }^{3}\right)$. Among the metric peculiarities of elliptic space which vitally affect the use of earlier methods are the following:

(A) Absence of free movability in the large. In the euclidean, hyperbolic, and spherical spaces the congruence of two subsets implies that the two subsets are superposable (that is, the existence of a congruent transformation of the space on itself which maps one subset on to the other) $\left({ }^{4}\right)$. In elliptic spaces this is not the case. If, for example, $p, q, s \in S_{2, r}$ with $p q=6 \pi r / 18, q s=7 \pi r / 18$, $p s=8 \pi r / 18$, and $p^{\prime}, q^{\prime}, s^{\prime} \in S_{2, r}$ with $p^{\prime} q^{\prime}=p q, q^{\prime} s^{\prime}=q s, p^{\prime} s^{\prime}=10 \pi r / 18$, then, upon identification of diametral point-pairs, the two triples are congruent subsets of the elliptic plane $E_{2, r}$ which, it turns out, are not superposable.

A simpler (and more disturbing) example of the nonvalidity of Euclid I.8 in elliptic space is furnished by the two triples $p, q, s$ and $p^{\prime}, q^{\prime}, s^{\prime}$ for which the spherical distances are $p q=q s=p s=\pi r / 3$ and $p^{\prime} q^{\prime}=q^{\prime} s^{\prime}=\pi r / 3, p^{\prime} s^{\prime}$ $=2 \pi r / 3$. Passing to the elliptic plane, the two triples are congruent but clearly not superposable, for $p^{\prime}, q^{\prime}, s^{\prime}$ lie on an elliptic line and $p, q, s$ do not. The property of free movability in the large is used extensively in obtaining characterizations of euclidean, hyperbolic, and spherical spaces and subsets.

(B) Distinction between "contained in" and "congruently contained in" for subsets of elliptic space. The example given in (A) of three points on an elliptic line congruent with a triple not on a line has no counterpart in the spaces previously studied. So far as the elliptic line is concerned, the difficulty brought about by this circumstance is not serious, for it may be shown that four points of a line are not congruent with four points of an elliptic space which are not contained in a line. But for higher-dimensional elliptic spaces the situa-

(2) The distance $p q$ of two points $p, q$ of $S_{n, r}$ is the length of the shorter arc of the great circle joining them.

(3) For a comparison of space and subset problems see [4, pp. 322-323].

(4) Since every congruent transformation of the elliptic plane on itself is a motion (a rotation), the term "superposable" seems a suitable one. 
tion cannot be brought back to normal by considering sets containing "enough" points; for the writer has proved that for every integer $k>3$, the elliptic plane, for example, contains $k$ points congruent with an elliptic subset of $k$ points not contained in any plane. An instructive example, referred to later, is furnished by six points of the plane $E_{2, r}$ which are congruent to six points contained in an elliptic five-space $E_{5, r}$ and not in any lower-dimensional subspace.

(C). Ordinary notions of dependence and independence not congruence invariants. The six points mentioned in (B) which lie in $E_{5, r}$, but not in $E_{k, r}$, $k<5$, would ordinarily be called a linearly independent set, but they are congruent with six points of $E_{2, r}$, a linearly dependent set. There is no possibility of ascertaining the lowest-dimensional subspace containing an elliptic subset merely from the mutual distances of the points of the set. This points to the difficulty of defining notions of metric dependence and independence which will (i) lead readily to metric definitions of the linear subspaces and (ii) disturb as little as possible the definitions of these notions already in use in other $\operatorname{spaces}\left({ }^{5}\right)$.

(D). The nonlinearity of the equidistant locus. Closely connected with (A) is the fact that in the elliptic plane the locus of points equidistant from two distinct points consists of two (mutually perpendicular) lines. This circumstance affects the character of a metric basis for the elliptic plane, since no set contained in two such lines can form a basis. In particular, no three points of $E_{2, r}$ form a metric basis for the plane.

(E). Equilateral subsets. Unlike the euclidean and hyperbolic planes (which contain no equilateral quadruples) and the spherical two-space (in which all equilateral quadruples are congruent and no equilateral quintuples exist) the elliptic plane contains (i) two (noncongruent) kinds of such quadruples, (ii) a unique class of equilateral quintuples and even a class of equilateral sextuples. The edge of an equilateral sextuple is $r \cdot \cos ^{-1}\left(1 / 5^{1 / 2}\right)$. It follows that though the euclidean, hyperbolic, and spherical two-spaces each have congruence order 5 with respect to semimetric spaces (that is, any semimetric space is congruently contained in one of these spaces provided each five of its points are) the least possible congruence order of the elliptic plane with respect to semimetric spaces is 7 . A complete analysis of equilateral subsets of $E_{n, r}$ has not yet been made for $n>2$.

In addition to a consideration of properties (A)-(E), we shall make much use of the following two results:

Result 1. A semimetric m-tuple $p_{1}, p_{2}, \cdots, p_{m}$ is congruently imbeddable in the elliptic space $E_{n, r}$ if and only if (i) $0 \leqq p_{i} p_{j} \leqq \pi r / 2(i, j=1,2, \cdots, m)$

${ }^{(5)}$ To meet these requirements the writer has formulated notions of "class dependence" and "relative dependence" with respect to a class of matrices. Since (c) plays a minor rôle in the developments of this paper, there is no need to discuss those concepts here. They have proved to be quite useful in the subset problem, where the difficulty seems fundamental. 
and (ii) there exists a symmetric square matrix $\epsilon=\left(\epsilon_{i j}\right), \epsilon_{i j}=\epsilon_{j i}= \pm 1, \epsilon_{i i}=1$ $(i, j=1,2, \cdots, m)$ such that the determinant $\left|\epsilon_{i j} \cos \left(p_{i} p_{j} / r\right)\right|(i, j=1,2$, $\cdots, m)$ has rank not exceeding $n+1$, with all nonvanishing principal minors positive.

A matrix $\left(\epsilon_{i j}\right)$, all of whose elements are \pm 1 , with the diagonal elements 1 , is referred to as an $\epsilon$-matrix.

Result 2. Two congruent triples $p_{1}, p_{2}, p_{3}$ and $p_{1}^{\prime}, p_{2}^{\prime}, p_{3}^{\prime}$ of the elliptic plane $E_{2, r}$ are superposable if (i) one of the distances $p_{i} p_{j}(i, j=1,2,3)$ equals $\pi r / 2$ or (ii) the determinant $\Delta^{*}\left(p_{1}, p_{2}, p_{3} ; r\right)=\left|\epsilon_{i j} \cos \left(p_{i} p_{j} / r\right)\right|(i, j=1,2,3)$ is negative, where every $\epsilon_{i j}=1$, except $\epsilon_{23}=-1$.

The proof of Result 1 appears elsewhere [4, pp. 335-336]. The second result is a special case of an unpublished theorem concerning criteria for the superposability of two congruent subsets of $E_{n, r .}$. The proof of this second result is, however, immediate; for if $\Delta^{*}<0$ then, by Result 1 , the determinant $\Delta\left(p_{1}, p_{2}, p_{3} ; r\right)=\left|\cos \left(p_{i} p_{j} / r\right)\right|(i, j=1,2,3)$ is non-negative and hence the elliptic distances $p_{i} p_{j}(i, j=1,2,3)$ are also spherical distances on $S_{2, r}$. The two congruent elliptic triples are then congruent spherical triples, and a congruent transformation of $S_{2, r}$ on itself exists which superposes them. This transformation is evidently a congruent mapping of the $E_{2, r}$ on itself ( $\left.{ }^{6}\right)$. The reader may easily convince himself of the superposability of the triples in case one of the distances is $\pi r / 2$.

The condition $\Delta^{*}<0$ is used in this paper to give a precise meaning to such phrases as "triples whose diameters are smaller than a fixed number, depending on the space," "points sufficiently close together," and so on, which are frequently encountered in articles on elliptic space. The requirement is reducible (upon expansion of the determinant) to the inequality $p_{1} p_{2}+p_{2} p_{3}$ $+p_{1} p_{3}<\pi r$, but the determinant form is retained because of its usefulness in suggesting extensions to more general cases.

3. Postulates for a $\Sigma_{r}$ space and preliminary theorems. A system $\{M ; r\}$ of a point set $M$, containing at least two points, and a positive number $r$ forms a $\Sigma_{r}$ space provided the following postulates are satisfied:

Postulate I. $\Sigma_{r}$ is semimetric (that is, there is associated with each pair of points $p, q$ of $M$, independent of order, a non-negative real number $p q$, called their distance, which is zero if and only if $p$ and $q$ are identical).

Postulate II. The diameter of $\Sigma_{r}$ is at most $\pi r / 2$ (that is, $p, q \in \Sigma_{r}$ implies $p q \leqq \pi r / 2)$.

Postulate III. Let $p_{0}, p_{1}, \cdots, p_{4}$ be any five pairwise distinct points of

(8) The condition $\Delta^{*}<0$ is sufficient for superposability of triples, but not necessary. Thus two triples with $p_{i} p_{j}=p_{i}^{\prime} p_{j}^{\prime}=4 \pi r / 9(i, j=1,2,3 ; i \neq j)$ may be superposable although $\Delta^{*}>0$. 
$\Sigma_{r}$ with (i) two triples linear and (ii) the determinant $\Delta^{*}$ formed for three of the points (one of the points being common to the two linear triples) negative. Then there exists an $\epsilon$-matrix such that all principal minors of $\left|\epsilon_{i j} \cos \left(p_{i} p_{i} / r\right)\right|$ $(i, j=0,1, \cdots, 4)$ are non-negative $\left.{ }^{7}\right)$.

Postulate IV. If $p, q$ are any two distinct points of $\Sigma_{r}$ with $p q \neq \pi r / 2$, points $d(p), d(q)$ exist such that $q$ is between $\left({ }^{8}\right) p$ and $d(p), p$ is between $q$ and $d(q)$ (that is, $p q d(p)$ and $q p d(q)$ subsist) and $p d(p)=q d(q)=\pi r / 2$.

Postulate V. $\Sigma_{r}$ is convex (that is, $\Sigma_{r}$ contains for each pair of distinct points at least one between point).

Postulate VI. $\Sigma_{r}$ is complete (that is, if $\left\{p_{i}\right\}$ is an infinite sequence of points of $\Sigma_{r}$ such that $p_{i} p_{j} \rightarrow 0$ as $i, j \rightarrow \infty$, then $\Sigma_{r}$ contains a point $p$ with $\lim _{i \rightarrow \infty} p p_{i}=0$ ).

Our object is (i) to show that $\Sigma_{r}$ is congruent with an elliptic space of finite or infinite dimension and space constant $r$ and (ii) to impose the necessary and sufficient additional metric conditions on $\Sigma_{r}$ which insure congruence with (a) the elliptic space $E_{n, r}$ of given dimension $n$ and (b) the elliptic space $E_{\infty, r}$ of infinite dimension, which arises upon identifying diametral pointpairs of the sphere of radius $r$ in Hilbert space.

THEOREM 1. If $p_{0}, p_{1}, \cdots, p_{4}$ are points of $\Sigma_{r}$ which satisfy the conditions of Postulate III, they are congruent with five points of the elliptic plane $E_{2, r}$.

Proof. By Postulate III and Result 1,

$$
p_{0}, p_{1}, \cdots, p_{4} \approx p_{0}^{\prime}, p_{1}^{\prime}, \cdots, p_{4}^{\prime},
$$

with the "primed" points in $E_{4, r}$. Since the five points of $\Sigma_{r}$ contain two linear triples, the two corresponding triples of the elliptic quintuple lie on elliptic lines $\left({ }^{9}\right)$ and hence $p_{0}^{\prime}, p_{1}^{\prime}, \cdots, p_{4}^{\prime}$ lie in an elliptic plane $E_{2, r}$.

Remark. An algebraic proof is obtained by showing that the determinant $\left|\epsilon_{i j} \cos \left(p_{i} p_{j} / r\right)\right|(i, j=0,1, \cdots, 4)$, which has every principal minor nonnegative (Postulate III) has rank not greater than 3. This is accomplished by noting that (i) the two third-order principal minors corresponding to the

(7) A triple is linear provided it is congruent with three points of the euclidean line; that is, the sum of two of the three distances determined by the (not necessarily pairwise distinct) points equals the third distance.

Postulate III has a local-global character, the local nature being derived from the condition that $\Delta^{*}$ be negative for three of the five points.

(8) A point $q$ is between points $p$ and $s$ provided $p \neq q \neq s, p q+q s=p s$. We symbolize this by $p q s$. A point diametral to $p$ is denoted by $d(p)$; that is, $p d(p)=\pi r / 2$.

Postulate IV gives the kind of external convexity needed.

(9) For the above proof it is important to remark that points of an elliptic space congruent with a linear triple necessarily lie on an elliptic line. This is not the case for points congruent with points of $\Sigma_{r}$ which are merely congruently contained in an elliptic line (see (b), §2). 
two linear triples are zero (for any allowable choice of the epsilons) and (ii) each fourth-order principal minor with a vanishing third-order principal minor is zero.

THEOREM 2. Each four pairwise distinct points of $\Sigma_{r}$ containing a linear triple is congruent with four points of $E_{2, r}$.

Proof. Let $q_{0}, q_{1}, q_{2}, q_{3}$ be such a quadruple with the labelling selected so that $q_{0} q_{1} q_{2}$ holds. Then each of the distances $q_{0} q_{1}, q_{1} q_{2}$ is less than $\pi r / 2$.

Case 1. The point $q_{3}$ is not between $q_{0}$ and $q_{1}$. By Postulate $\mathrm{V}$ there is a point $q_{4}$ such that $q_{0} q_{4} q_{1}$ holds. Then $q_{4}$ is distinct from $q_{i}(i=0,1,2,3)$ $\left(q_{4} \neq q_{2}\right.$ since $q_{0} q_{4}<q_{0} q_{1}<q_{0} q_{2}$, and $q_{4} \neq q_{3}$ since $q_{4}$ is between $q_{0}, q_{1}$ while $q_{3}$ is not) and the five pairwise distinct points $q_{0}, q_{1}, \cdots, q_{4}$ satisfy the conditions of Theorem 1, since the triples $q_{0}, q_{1}, q_{2}$ and $q_{0}, q_{1}, q_{4}$ are linear, while $\Delta^{*}\left(q_{0}, q_{1}, q_{4} ; r\right)$ is negative (for since $q_{0} q_{4}+q_{4} q_{1}=q_{0} q_{1}<\pi r / 2$ it is clear that $q_{0} q_{4}+q_{4} q_{1}+q_{0} q_{1}<\pi r$ ). Hence $q_{0}, q_{1}, \cdots, q_{4}$ (and consequently $q_{0}, q_{1}, q_{2}, q_{8}$ ) are imbeddable in $E_{2, r}$.

Case 2. The point $q_{3}$ is between $q_{0}$ and $q_{1}$. Let $q_{4}$ be a point of $\Sigma_{r}$ between $q_{0}$ and $q_{3}$. Then $q_{4} \neq q_{0}, q_{1}, q_{2}, q_{3}\left(q_{4} \neq q_{1}\right.$ since $q_{0} q_{4}<q_{0} q_{3}<q_{0} q_{1}$ and $q_{4} \neq q_{2}$ since $\left.q_{0} q_{4}<q_{0} q_{1}<q_{0} q_{2}\right)$ and the pairwise distinct points $q_{0}, q_{1}, \cdots, q_{4}$ satisfy the conditions of Theorem 1 since $q_{0}, q_{1}, q_{2}$ and $q_{0}, q_{3}, q_{4}$ are linear triples and $q_{0} q_{3}+q_{3} q_{4}+q_{0} q_{4}=2 q_{0} q_{3}<2 q_{0} q_{1}<\pi r$ implies that the determinant $\Delta^{*}\left(q_{0}, q_{3}, q_{4} ; r\right)$ is negative. We conclude that $q_{0}, q_{1}, q_{2}, q_{3}$ are imbeddable in $E_{2, r}\left({ }^{10}\right)$.

THEOREM 3. Each triple of points of $\Sigma_{r}$ is congruently contained in $E_{2, r}$.

Proof. Using Postulate II in the contrary case, suppose $q_{0}, q_{1}, q_{2}$ pairwise distinct. If the triple is linear it is evidently imbeddable in the elliptic line $E_{1, r} ;$ if the triple is not linear, let $q_{3}$ be a point of $\Sigma_{r}$ between $q_{1}$ and $q_{2}$ (Postulate V). Then $q_{0}, q_{1}, q_{2}, q_{3}$ are four pairwise distinct points $\left(q_{3} \neq q_{0}\right.$ since $q_{1}, q_{2}, q_{3}$ are linear while $q_{0}, q_{1}, q_{2}$ are not) containing a linear triple and hence (Theorem 2) are imbeddable in $E_{2, r}$.

Since $E_{2, r}$ is a metric space, we have:

COROLlary. The space $\Sigma_{r}$ is a metric space.

4. Segments and lines of $\Sigma_{r}$. In this section the elliptic character of certain sets of lines of $\Sigma_{r}$ is established, and properties of the space are obtained which serve to anchor the induction to be employed in $\$ 5$.

THEOREM 4. Two distinct non-diametral points of $\Sigma_{r}$ are joined by exactly one segment $\left({ }^{11}\right)$.

(10) Since $\Sigma_{r}$ has not yet been proved metric, properties of betweenness peculiar to metric spaces (which would greatly simplify the proof of Theorem 2) cannot be used.

(11) A simple arc joining two points $p, q$ is called a segment provided it is congruent with a straight line segment of length $p q$. 
Proof. Since $\Sigma_{r}$ is complete, convex and metric each pair of its (distinct) points is joined by at least one segment [5, pp. 87-89](12). If $p, q \in \Sigma_{r}$ $(0<p q<\pi r / 2)$ which are joined by two distinct segments then a constant $c$ $(0<c<1)$ exists such that the relations $p x+x q=p q, p x=c \cdot p q$ are satisfied by two distinct points $a, b$ of $\Sigma_{r}$. The four pairwise distinct points $p, q, a, b$ are (Theorem 2) congruent with $p^{\prime}, q^{\prime}, a^{\prime}, b^{\prime}$ of $E_{2, r}$ and since $p^{\prime} a^{\prime} q^{\prime}$ and $p^{\prime} b^{\prime} q^{\prime}$ hold, the "primed" points lie on an elliptic line. But an elliptic line does not contain two distinct points with the same distances from two distinct non-diametral points of the line, and hence $a^{\prime}=b^{\prime}$. Then $a=b$, a contradiction which establishes the theorem.

The unique segment joining two distinct non-diametral points $p, q$ of $\Sigma_{r}$ is denoted by seg. $(p, q)$.

Corollary. The congruence $p, q \approx p^{\prime}, q^{\prime}\left(p^{\prime}, q^{\prime} \in E_{n, r}\right), p \neq q$, determines uniquely the congruence seg. $(p, q) \approx \operatorname{seg} .\left(p^{\prime}, q^{\prime}\right)$ (that is, there is one and only one congruence between the two segments which maps $p, q$ onto $p^{\prime}, q^{\prime}$, respectively).

If $p q d(p)$ holds $(p d(p)=\pi r / 2)$ then $p q<\pi r / 2, q d(p)<\pi r / 2$, and unique segments seg. $(p, q)$, seg. $(q, d(p))$ are determined which have only $q$ in common (for $p q d(p)$ and $p x q$ imply (since $\Sigma_{r}$ is metric) $x q d(p)$, and so $x \bar{E}$ seg. $(q, d(p))$. Because $p q d(p)$ holds and $\Sigma_{r}$ is complete, convex, and metric, seg. $(p, q)$ can be prolonged to $d(p)$, and since only one segment joins $q$ and $d(p)$, it follows that the sum seg. $(p, q)+$ seg. $(q, d(p))$ is a segment $[3$, p. 47]. Thus diametral point-pairs $p, d(p)$ are joined by segments (of length $\pi r / 2$ ) and if $p q d(p)$ holds there is only one seg. $(p, q, d(p))$.

TheOREM 5. If $p, q \in \Sigma_{r}(0<p q<\pi r / 2)$ there is exactly one point $d(p)$ of $\Sigma_{r}$ with $p q d(p)$.

Proof. By Postulate IV at least one point with this property exists. If $d_{1}(p), d_{2}(p)$ are two distinct points with $p q d_{1}(p), p q d_{2}(p)$ then the four pairwise distinct points are congruent with $p^{\prime}, q^{\prime}, d_{1}\left(p^{\prime}\right), d_{2}\left(p^{\prime}\right)$ of $E_{2, r}$. Then $q^{\prime}$ belongs to both elliptic lines $E_{1, r}\left(p^{\prime}, d_{1}\left(p^{\prime}\right)\right), E_{1, r}\left(p^{\prime}, d_{2}\left(p^{\prime}\right)\right)$, which are distinct since $d_{1}\left(p^{\prime}\right) \neq d_{2}\left(p^{\prime}\right)$, and hence $p^{\prime}=q^{\prime}$, which contradicts $p^{\prime} q^{\prime}=p q>0$.

If, now, $p, q \in \Sigma_{r}(0<p q<\pi r / 2)$ consider the four pairwise distinct points $p, q, d(p), d(q)$, where $d(p), d(q)$ are the unique and evidently distinct points of $\Sigma_{r}$ such that $p q d(p), q p d(q)$ hold. Since points $p^{\prime}, q^{\prime}, d\left(p^{\prime}\right), d\left(q^{\prime}\right)$ of $E_{1, r}$ exist congruent with $p, q, d(p), d(q)$ and each point of an elliptic line, distinct from each point of a diametral point-pair, is a between point of the pair, the relations $p d(q) d(p)$ and $q d(p) d(q)$ hold. No one of the distances $p q, q d(p)$, $d(p) d(q), d(q) p$ equals $\pi r / 2$, and so the four points determine the unique segments seg. $(p, q)$, seg. $(q, d(p))$, seg. $(d(p), d(q))$, seg. $(d(q), p)$, which, as is easily seen, have pairwise at most end points in common.

(12) See also [3, pp. 40-41]. 
From $p q d(p)$ and $p d(q) d(p)$ it follows that

$$
\begin{gathered}
\text { seg. }(p, q)+\text { seg. }(q, d(p))=\text { seg. }(p, q, d(p)), \\
\text { seg. }(p, d(q))+\text { seg. }(d(q), d(p))=\text { seg. }(p, d(q), d(p)),
\end{gathered}
$$

the two segment-sums having only the points $p, d(p)$ in common.

Definition. If $p, q \in \Sigma_{r}(0<p q<\pi r / 2)$, then the sum seg. $(p, q, d(p))$ +seg. $(p, d(q), d(p))$, where $d(p), d(q)$ are the unique points diametral to $p, q$, respectively, such that $p q d(p), q p d(q)$ hold, is called a one-dimensional subspace $\Sigma_{1, r}$ of $\Sigma_{r}$, with base points $p, q$.

Since the preceding discussion shows that $\Sigma_{1, r}$ is uniquely determined by base points $p, q$, we may denote it by $\Sigma_{1, r}(p, q)$. Note that $\Sigma_{1, r}$ is a simple closed curve.

THEOREM 6. $A$ one-dimensional subspace $\Sigma_{1, r}$ of $\Sigma_{r}$ is congruent with the elliptic line $E_{1 . r .}$.

Proof. If we denote (non-diametral) base points of $\Sigma_{1, r}$ by $p, q, \Sigma_{1, r}(p, q)$ $=$ seg. $(p, q, d(p))+$ seg. $(p, d(q), d(p))$. The points $p^{\prime}, q^{\prime}, d\left(p^{\prime}\right), d\left(q^{\prime}\right)$ of $E_{2, r}$ congruent with $p, q, d(p), d(q)$ lie, as already observed, in a line $E_{1, r}\left(p^{\prime}, q^{\prime}\right)$, and clearly

$$
\begin{gathered}
\text { seg. }(p, q, d(p)) \approx \text { seg. }\left(p^{\prime}, q^{\prime}, d\left(p^{\prime}\right)\right), \\
\text { seg. }(p, d(q), d(p)) \approx \operatorname{seg} .\left(p^{\prime}, d\left(q^{\prime}\right), d\left(p^{\prime}\right)\right),
\end{gathered}
$$

with $E_{1, r}=$ seg. $\left(p^{\prime}, q^{\prime}, d\left(p^{\prime}\right)\right)+$ seg. $\left(p^{\prime}, d\left(q^{\prime}\right), d\left(p^{\prime}\right)\right)$.

These two congruences establish a mapping of $\Sigma_{1, r}(p, q)$ onto $E_{1, r}\left(p^{\prime}, q^{\prime}\right)$. To show the mapping a congruent one, suppose $x, y \in \Sigma_{1, r}$ mapped on elements $x^{\prime}, y^{\prime}$, respectively, of $E_{1, r}$. If $x, y$ are both in the same component segment then $x y=x^{\prime} y^{\prime}$ by one of the above congruences. In the contrary case, suppose $x \in$ seg. $(p, q, d(p)), y \in$ seg. $(p, d(q), d(p))$, and let $p_{1}, p_{2}$ be points of the first and second of these segments, respectively, distinct from $q, d(q), x, y$, such that $p p_{1} q, p p_{2} d(q)$ hold and $\Delta^{*}\left(p, p_{1}, p_{2} ; r\right)<0$, with $p_{1}^{\prime}, p_{2}^{\prime \prime}$ the points corresponding to $p_{1}, p_{2}$ by means of congruences (1), (2), respectively $\left({ }^{18}\right)$. The pairwise distinct points $p, p_{1}, p_{2}, q, d(q)$ satisfy the conditions of Theorem 1 and consequently are congruent with points $p^{\prime \prime}, p_{1}^{\prime \prime}, p_{2}^{\prime \prime}, q^{\prime \prime}, d\left(q^{\prime \prime}\right)$ of $E_{2, r}$. Since these points necessarily lie on an elliptic segment with end points $q^{\prime \prime}, d\left(q^{\prime \prime}\right)$, the points $p^{\prime \prime}, p_{1}^{\prime \prime}, p_{2}^{\prime \prime}$ are linear. Hence $p, p_{1}, p_{2}$ are linear, $p_{1} p_{2}=p_{1}^{\prime \prime} p_{2}^{\prime \prime}=p_{1}^{\prime \prime} p^{\prime \prime}+p^{\prime \prime} p_{2}^{\prime \prime}=p_{1} p+p p_{2}=p_{1}^{\prime \prime} p^{\prime}+p^{\prime} p_{2}^{\prime}=p_{1}^{\prime} p_{2}^{\prime}$ and so $p, p_{1}, p_{2}$ $\approx p^{\prime}, p_{1}^{\prime}, p_{2}^{\prime}$.

If we apply Theorem 1 to $p, p_{1}, p_{2}, x, y$ (the triples $p, p_{1}, x$ and $p, p_{2}, y$ are linear)

$$
p, p_{1}, p_{2}, x, y \approx p^{\prime \prime}, p_{1}^{\prime \prime}, p_{2}^{\prime \prime}, x^{\prime \prime}, y^{\prime \prime}, \text { of } E_{2, r}
$$

(13) Since $\Delta^{*}\left(p, p_{1}, p_{2} ; r\right)<0$ whenever $p p_{1}+p p_{2}+p_{1} p_{2}<\pi r$, it is clear that points $p_{1}, p_{2}$ with $\max \left(p p_{1}, p p_{2}\right)<\min (p q, p d(q), \pi r / 6)$ satisfy all conditions. 
(the "double-primed" points not necessarily the same as those above). Since $p^{\prime \prime}, p_{1}^{\prime \prime}, p_{2}^{\prime \prime} \approx p, p_{1}, p_{2} \approx p^{\prime}, p_{1}^{\prime}, p_{2}^{\prime}$ and $\Delta^{*}\left(p, p_{1}, p_{2} ; r\right)<0$, a congruent transformation of $E_{2, r}$ onto itself exists (Result 2) mapping $p^{\prime \prime}, p_{1}^{\prime \prime}, p_{2}^{\prime \prime}$ onto $p^{\prime}, p_{1}^{\prime}, p_{3}^{\prime}$, respectively. This transformation maps $x^{\prime \prime}$ on a point $x^{*}$ of the line $E_{1, r}\left(p^{\prime}, p_{1}^{\prime}\right)=E_{1, r}\left(p^{\prime}, q^{\prime}\right)$ such that $p^{\prime} x^{*}=p^{\prime \prime} x^{\prime \prime}=p x=p^{\prime} x^{\prime}$ and $p_{1}^{\prime} x^{*}$ $=p_{1}^{\prime \prime} x^{\prime \prime}=p_{1} x=p_{1}^{\prime} x^{\prime}$, that is, $x^{*}$ has the same distances as $x^{\prime}$ from the nondiametral points $p^{\prime}, p_{1}^{\prime}$ of $E_{1, r}\left(p^{\prime}, q^{\prime}\right)$ and hence $x^{*}=x^{\prime}$. Similarly $y^{\prime \prime}$ is mapped on $y^{\prime}, p, p_{1}, p_{2}, x, y \approx p^{\prime}, p_{1}^{\prime}, p_{2}^{\prime}, x^{\prime}, y^{\prime}$ and so $x y=x^{\prime} y^{\prime}$.

Remark. A different method obtains the congruence of $\Sigma_{1, r}$ and $E_{1, r}$ by means of Theorem 2. Thus in a space whose postulates are those of $\Sigma_{r}$ except that Postulate III is replaced by the weaker demand of Theorem 2, onedimensional subspaces (defined exactly as above) are congruent with elliptic lines.

\section{COROllary. Each one-dimensional subspace $\Sigma_{1, r}$ has length $\pi r$.}

LEmma. If $s, t \in \Sigma_{1, r}(0<s t<\pi r / 2)$ then $\operatorname{seg} .(s, t) \subset \Sigma_{1, r}$.

Proof. By Theorem $6, \Sigma_{1, r} \approx E_{1, r}$ and the points $s^{\prime}, t^{\prime}$ of $E_{1, r}$ congruent to $s, t$ determine the elliptic segment seg. $\left(s^{\prime}, t^{\prime}\right)$ which is a subset of $E_{1, r .}$. Since a congruence transforms segments into segments, the subset of $\Sigma_{1, r}$ congruent to seg. $\left(s^{\prime}, t^{\prime}\right)$ by the above congruence is a segment with end points $s, t$ and hence (Theorem 4$)$ is seg. $(s, t)$.

LEMMA. $A$ diametral point-pair of $\Sigma_{r}$ is contained in one and only one subspace $\Sigma_{1, r}$.

Proof. If $p, d(p)$ is such a pair and $q$ any point of $\Sigma_{r}$ between $p$ and $d(p)$, the unique subspace $\Sigma_{1, r}(p, q)$ contains $p$ and $d(p)$. Let $\Sigma_{1, s}^{*}$ be any one-dimensional subspace containing $p, d(p)$. If $x \in \Sigma_{1, r}^{*}$ then the congruence of $\Sigma_{1, r}^{*}$ with an elliptic line shows that $p x d(p)$ holds. Select points $p_{1}, p_{2}$ of $\Sigma_{1, r}(p, q)$ (distinct from $p, x)$ such that $p_{1} \in$ seg. $(p, q, d(p)), p_{2} \in$ seg. $(p, d(q), d(p))$, and $0<p p_{1}=p p_{2}<\pi r / 6$.

By Theorem $1, p, p_{1}, p_{2}, d(p), x \approx p^{\prime}, p_{1}^{\prime}, p_{2}^{\prime}, d\left(p^{\prime}\right), x^{\prime}$, points of $E_{2, r}$, and it is clear (since $p^{\prime} x^{\prime} d\left(p^{\prime}\right)$ holds) that the "primed" points all lie on a line $E_{1, r}$ Hence $x^{\prime}$ belongs to one of the segments seg. $\left(p^{\prime}, p_{1}^{\prime}\right)$, seg. $\left(p_{1}^{\prime}, d\left(p^{\prime}\right)\right)$, seg. $\left(d\left(p^{\prime}\right), p_{2}^{\prime}\right)$, seg. $\left(p^{\prime}, p_{2}^{\prime}\right)$ and so $x$ is a point of one of the segments seg. $\left(p, p_{1}\right)$, seg. $\left(p_{1}, d(p)\right)$, seg. $\left(d(p), p_{2}\right)$, seg. $\left(p, p_{2}\right)$. From the preceding lemma, $x \in \Sigma_{1, r}(p, q)$ and $\Sigma_{1, r}^{*} \subset \Sigma_{1, r}(p, q)$. Since each subspace is congruent with $E_{1, r}$ it follows that $\Sigma_{1, r}^{*}=\Sigma_{1, r}(p, q)$ and the lemma is proved.

LEMma. $A$ non-diametral point-pair is contained in one and only one subspace $\Sigma_{1, r \cdot}$

Proof. Each subspace $\Sigma_{1, r}$ containing the non-diametral points $p, q$ evidently contains the unique point $d(p)$ such that $p q d(p)$ holds, and the lemma follows from the preceding one. 
Combining these lemmas, we have:

THEOREM 7. There is one and only one subspace $\Sigma_{1, r}$ containing any given pair of distinct points of $\Sigma_{r}$.

Thus a one-dimensional subspace contains (is identical with) the onedimensional subspace determined by any two of its points. We refer to $\Sigma_{1, \text { r }}$ as a line of $\Sigma_{r}$.

COROLlaRY 1. Two distinct lines of $\Sigma_{r}$ have at most one point in common.

Corollary 2. Any two non-diametral points of a one-dimensional subspace may be taken as base-points of the subspace.

THEOREM 8. Let $p_{0}, p_{1}, p_{2}$ be three pairwise distinct points of $\Sigma_{r}$ with $\Delta^{*}\left(p_{0}, p_{1}, p_{2} ; r\right)<0$. If $p_{0}^{\prime}, p_{1}^{\prime}, p_{2}^{\prime}$ are any points of the elliptic plane, with $p_{0}, p_{1}, p_{2} \approx p_{0}^{\prime}, p_{1}^{\prime}, p_{2}^{\prime}$, then the congruences

$$
\Sigma_{1, r}\left(p_{0}, p_{1}\right) \approx E_{1, r}\left(p_{0}^{\prime}, p_{1}^{\prime}\right),
$$

$$
\Sigma_{1, r}\left(p_{0}, p_{2}\right) \approx E_{1, r}\left(p_{0}^{\prime}, p_{2}^{\prime}\right)
$$

determine uniquely the congruence

$$
\Sigma_{1, r}\left(p_{0}, p_{1}\right)+\Sigma_{1, r}\left(p_{0}, p_{2}\right) \approx E_{1, r}\left(p_{0}^{\prime}, p_{1}^{\prime}\right)+E_{1, r}\left(p_{0}^{\prime}, p_{2}^{\prime}\right) .
$$

Proof. Since $\Delta^{*}\left(p_{0}, p_{1}, p_{2} ; r\right)<0$ then the determinant $\Delta\left(p_{0}, p_{1}, p_{2} ; r\right)$ $=\left|\cos \left(p_{i} p_{j} / r\right)\right|(i, j=0,1,2)$ is non-negative (Result 1$)$ and it follows that no one of the distances $p_{i} p_{i}(i, j=0,1,2)$ is $\pi r / 2$. Hence $p_{0}, p_{1}$ and $p_{0}, p_{2}$ may be taken as base points of lines $\Sigma_{1, r}\left(p_{0}, p_{1}\right), \Sigma_{1, r}\left(p_{0}, p_{2}\right)$, respectively, of $\Sigma_{r}$. The notation of the congruences (a), (b) indicates that $p_{i}$ and $p_{i}^{\prime}$ $(i=0,1,2)$ are corresponding points, and since $p_{0} p_{i} \neq \pi r / 2(i=1,2)$, these congruences are unique. If $\Delta\left(p_{0}, p_{1}, p_{2} ; r\right)=0$, the points $p_{0}, p_{1}, p_{2}$ are linear, $p_{2} \in \Sigma_{1, r}\left(p_{0}, p_{1}\right)$ and so $\Sigma_{1, r}\left(p_{0}, p_{1}\right)=\Sigma_{1, r}\left(p_{0}, p_{2}\right)$ (Theorem 7). Similarly $E_{1, r}\left(p_{0}^{\prime}, p_{1}^{\prime}\right)=E_{1, r}\left(p_{0}^{\prime}, p_{2}^{\prime}\right)$ and the theorem follows from Theorem 6 .

If, on the other hand, $\Delta\left(p_{0}, p_{1}, p_{2} ; r\right)=\Delta\left(p_{0}^{\prime}, p_{1}^{\prime}, p_{2}^{\prime} ; r\right)$ is positive, then since $\Delta^{*}\left(p_{0}^{\prime}, p_{1}^{\prime}, p_{2}^{\prime} ; r\right)<0$, the points $p_{0}^{\prime}, p_{1}^{\prime}, p_{2}^{\prime}$ neither lie on an elliptic line nor are they congruent with points of such a line and consequently $p_{0}, p_{1}, p_{2}$ do not lie on a line of $\Sigma_{r}$. The congruences (a), (b), insured by Theorem 6 , give a mapping of the set $\Sigma_{1, r}\left(p_{0}, p_{1}\right)+\Sigma_{1, r}\left(p_{0}, p_{2}\right)$ onto the set $E_{1, r}\left(p_{0}^{\prime}, p_{1}^{\prime}\right)+E_{1, r}\left(p_{0}^{\prime}, p_{2}^{\prime}\right)$. To prove the mapping a congruent one it evidently suffices to examine the case in which $x \in \Sigma_{1, r}\left(p_{0}, p_{1}\right), y \in \Sigma_{1, r}\left(p_{0}, p_{2}\right)$ and $x^{\prime}, y^{\prime}$ are the points of the elliptic lines corresponding by congruences (a), (b).

Suppose, first, that $p_{0}, p_{1}, x$ and $p_{0}, p_{2}, y$ are both linear triples (that is, $x$ and $y$ are not interior points of the segments seg. $\left(d_{1}\left(p_{0}\right), d_{1}\left(p_{1}\right)\right)$, seg. $\left(d_{2}\left(p_{0}\right), d_{2}\left(p_{2}\right)\right)$, respectively, where $d_{1}\left(p_{0}\right), d_{1}\left(p_{1}\right)$ are points of $\Sigma_{1, r}\left(p_{0}, p_{1}\right)$ which are diametral to $p_{0}$ and $p_{1}$, respectively, and $d_{2}\left(p_{0}\right), d_{2}\left(p_{2}\right)$ are similarly defined points of $\Sigma_{1, r}\left(p_{0}, p_{2}\right)$, with $x \neq p_{0}, p_{1}$ and $y \neq p_{0}, p_{2}$. The points 
$p_{0}, p_{1}, p_{2}, x, y$ satisfy the conditions of Theorem 1 and hence

$$
p_{0}, p_{1}, p_{2}, x, y \approx p_{0}^{\prime \prime}, p_{1}^{\prime \prime}, p_{2}^{\prime \prime}, x^{\prime \prime}, y^{\prime \prime} \text { of } E_{2, r} \text {. }
$$

Since $p_{0}^{\prime \prime}, p_{1}^{\prime \prime}, p_{2}^{\prime \prime} \approx p_{0}, p_{1}, p_{2} \approx p_{0}^{\prime}, p_{1}^{\prime}, p_{2}^{\prime}$ and $\Delta^{*}\left(p_{0}, p_{1}, p_{2} ; r\right)<0$, a congruent transformation of $E_{2, r}$ on itself exists mapping $p_{i}^{\prime \prime}$ onto $p_{i}^{\prime}(i=0,1,2)$. Points $p_{0}, p_{1}, x$ being linear, so are $p_{0}^{\prime \prime}, p_{1}^{\prime \prime}, x^{\prime \prime}$ and consequently they lie on an elliptic line. As the transformation sends lines into lines, it maps $x^{\prime \prime}$ into the unique point $x^{*}$ of $\Sigma_{1, r}\left(p_{0}^{\prime}, p_{1}^{\prime}\right)$ with given distances $\left(p_{0} x, p_{1} x\right)$ from the non-diametral points $p_{0}, p_{1}$. Then $p_{0}^{\prime} x^{*}=p_{0}^{\prime \prime} x^{\prime \prime}=p_{0} x=p_{0}^{\prime} x^{\prime}$ and $p_{1}^{\prime} x^{*}=p_{1}^{\prime \prime} x^{\prime \prime}$ $=p_{1} x=p_{1}^{\prime} x^{\prime}$. Since both $x^{*}$ and $x^{\prime}$ are points of $E_{1, r}\left(p_{0}^{\prime}, p_{1}^{\prime}\right)$ it follows that $x^{*}=x^{\prime}$. In the same manner it is seen that $y^{\prime \prime}$ maps into $y^{\prime}$ and hence $p_{0}, p_{1}, p_{2}, x, y \approx p_{0}^{\prime}, p_{1}^{\prime}, p_{2}^{\prime}, x^{\prime}, y^{\prime}$, from which $x y=x^{\prime} y^{\prime}$.

Thus the set

$$
\begin{aligned}
\left\{\Sigma_{1, r}\left(p_{0}, p_{1}\right)-\text { int. seg. }\left(d_{1}\left(p_{0}\right), d_{1}\left(p_{1}\right)\right)\right\} & \\
+ & \left\{\Sigma_{1, r}\left(p_{0}, p_{2}\right)-\text { int. seg. }\left(d_{2}\left(p_{0}\right), d_{2}\left(p_{2}\right)\right)\right\}
\end{aligned}
$$

is congruently contained in $E_{1, r}\left(p_{0}^{\prime}, p_{1}^{\prime}\right)+E_{1, r}\left(p_{0}^{\prime}, p_{2}^{\prime}\right)\left({ }^{14}\right)$.

Let now $q_{1}, q_{2}$ be points of $\Sigma_{1, r}\left(p_{0}, p_{1}\right), \Sigma_{1, r}\left(p_{0}, p_{2}\right)$, respectively, with $0<p_{0} q_{1}<\epsilon, 0<p_{0} q_{2}<\epsilon\left(\epsilon>0\right.$, arbitrarily small) and let $q_{1}^{\prime}, q_{2}^{\prime}$ be the corresponding points on $E_{1, r}\left(p_{0}^{\prime}, p_{1}^{\prime}\right), E_{1, r}\left(p_{0}^{\prime}, p_{2}^{\prime}\right)$, respectively, by means of (a), (b). Then exactly as above

$$
\begin{aligned}
\left\{\Sigma_{1, r}\left(p_{0}, q_{1}\right)-\text { int. seg. }\left(d_{1}\left(p_{0}\right), d_{1}\left(q_{1}\right)\right)\right\} & \\
& +\left\{\Sigma_{1, r}\left(p_{0}, q_{2}\right)-\text { int. seg. }\left(d_{2}\left(p_{0}\right), d_{2}\left(q_{2}\right)\right)\right\}
\end{aligned}
$$

is congruently contained in $E_{1, r}\left(p_{0}^{\prime}, q_{1}^{\prime}\right)+E_{1, r}\left(p_{0}^{\prime}, q_{2}^{\prime}\right)$.

Since $\Sigma_{1, r}\left(p_{0}, q_{i}\right)=\Sigma_{1, r}\left(p_{0}, p_{i}\right)$ and $E_{1, r}\left(p_{0}^{\prime}, q_{i}^{\prime}\right)=E_{1, r}\left(p_{0}^{\prime}, p_{i}^{\prime}\right)(i=1,2)$ we see that with the exception of the interiors of two segments, each of arbitrarily small length $\epsilon$, the sum $\Sigma_{1, r}\left(p_{0}, p_{1}\right)+\Sigma_{1, r}\left(p_{0}, p_{2}\right)$ is congruently contained in the sum of the two corresponding elliptic lines. It follows by continuity that $\Sigma_{1, r}\left(p_{0}, p_{1}\right)+\Sigma_{1, r}\left(p_{0}, p_{2}\right)$ is congruently contained in $E_{1, r}\left(p_{0}^{\prime}, p_{1}^{\prime}\right)+E_{1, r}\left(p_{0}^{\prime}, p_{2}^{\prime}\right)$ and the mapping of the one set onto the given set, by congruences (a), (b), is a congruent one. The uniqueness of the congruence follows from that of the two defining congruences.

COROLlaRY 1. The sum of any two intersecting lines of $\Sigma_{r}$ is congruently imbeddable in $E_{2, r}$.

CoRollary 2. If $p_{0}, p_{1}, p_{2}$ are pairwise distinct points of $\Sigma_{r}$ with $\Delta^{*}\left(p_{0}, p_{1}, p_{2} ; r\right)<0$, and $p_{0}, p_{1}, p_{2} \approx p_{0}^{\prime}, p_{1}^{\prime}, p_{2}^{\prime}$, points of $E_{2, r}$, then the three congruences

$$
\Sigma_{1, r}\left(p_{i}, p_{i+1}\right) \approx E_{1, r}\left(p_{i}^{\prime}, p_{i+1}^{\prime}\right) \quad\left(i=0,1,2 ; p_{3}=p_{0}\right)
$$

(14) The cases $x=p_{1}, y=p_{2}$ are taken care of by the continuity of the metric. They may also be proved separately by use of Theorem 2 . 
determine uniquely the congruence

$$
\begin{aligned}
\Sigma_{1, r}\left(p_{0}, p_{1}\right)+\Sigma_{1, r}\left(p_{1}, p_{2}\right)+\Sigma_{1, r} & \left(p_{0}, p_{2}\right) \\
& \approx E_{1, r}\left(p_{0}^{\prime}, p_{1}^{\prime}\right)+E_{1, r}\left(p_{1}^{\prime}, p_{2}^{\prime}\right)+E_{1, r}\left(p_{0}^{\prime}, p_{2}^{\prime}\right) .
\end{aligned}
$$

Proof. The three congruences of the pairs of lines determine a mapping of the sum of the three lines of $\Sigma_{r}$ onto the sum of the corresponding three elliptic lines. By the preceding theorem the mapping defines uniquely a congruence between the sum of any two of the three lines of $\Sigma_{r}$ and the sum of the corresponding two lines of $E_{2, r}$. Since points $x, y$ of the sum of all three lines necessarily are contained in the sum of two of the lines, the corollary follows at once.

COROLLARY 3. Each set of five points of $\Sigma_{r}$ containing two linear triples is congruently imbeddable in $E_{2, r}$.

Proof. Each such set is evidently contained in two intersecting lines of $\boldsymbol{\Sigma}_{\mathbf{r}}$.

THEOREM 9. Let $p_{0}, p_{0,1}, p_{1}, p_{2}$ be pairwise distinct points of $\Sigma_{r}$ such that $p_{0,1} \in \Sigma_{1, r}\left(p_{0}, p_{1}\right), p_{2} \bar{E} \Sigma_{1, r}\left(p_{0}, p_{1}\right), p_{0} p_{2} \neq \pi r / 2$. If $p_{0}{ }^{\prime}, p_{0,1}^{\prime}, p_{1}{ }^{\prime}, p_{2}{ }^{\prime}$ are any points of $E_{2, r}$ such that $p_{0,1}^{\prime} \in E_{1, r}\left(p_{0}^{\prime}, p_{1}^{\prime}\right)$ and $p_{0}, p_{0,1}, p_{1}, p_{2} \approx p_{0}^{\prime}, p_{0,1}^{\prime}, p_{1}^{\prime}, p_{2}^{\prime}$, the congruences (a) $\Sigma_{1, r}\left(p_{0}, p_{2}\right) \approx E_{1, r}\left(p_{0}^{\prime}, p_{2}^{\prime}\right),(\mathrm{b}) \Sigma_{1, r}\left(p_{0}, p_{0,1}, p_{1}\right) \approx E_{1, r}\left(p_{0}^{\prime}, p_{0,1}^{\prime}, p_{1}^{\prime}\right)$ determine the unique congruence

$$
\Sigma_{1, r}\left(p_{0}, p_{0,1}, p_{1}\right)+\Sigma_{1, r}\left(p_{0}, p_{2}\right) \approx E_{1, r}\left(p_{0}^{\prime}, p_{0,1}^{\prime}, p_{1}^{\prime}\right)+E_{1, r}\left(p_{0}^{\prime}, p_{2}^{\prime}\right) .
$$

Proof. The points $p_{0}^{\prime}, p_{0,1}^{\prime}, p_{1}^{\prime}, p_{2}^{\prime}$ of $E_{2, r}$ are not on a line, for if so they would contain two linear triples, and hence a linear triple with $p_{2}^{\prime}$ as an element. But then $p_{2}$ forms with two of the points $p_{0}, p_{0,1}, p_{1}$ (which lie on a line) a linear triple and so $p_{2} \in \Sigma_{1, r}\left(p_{0}, p_{1}\right)$, contrary to hypothesis. Thus the lines $E_{1, r}\left(p_{0}^{\prime}, p_{0,1}^{\prime}, p_{1}^{\prime}\right), E_{1, r}\left(p_{0}^{\prime}, p_{2}^{\prime}\right)$ are distinct.

Let $x, y$ be elements of $\Sigma_{1, r}\left(p_{0}, p_{0,1}, p_{1}\right)+\Sigma_{1, r}\left(p_{0}, p_{2}\right)$ and $x^{\prime}, y^{\prime}$ the elements of $E_{1, r}\left(p_{0}^{\prime}, p_{0,1}^{\prime}, p_{1}^{\prime}\right)+E_{1, r}\left(p_{0}^{\prime}, p_{2}^{\prime}\right)$ corresponding to them in the mapping of the one sum onto the other given by congruences (a), (b). We may suppose $x$ a point of the first and $y$ a point of the second summand.

By Corollary 1 of the preceding theorem,

$$
p_{0}, p_{0,1}, p_{1}, p_{2}, x, y \approx p_{0}^{\prime \prime}, p_{0,1}^{\prime \prime}, p_{1}^{\prime \prime}, p_{2}^{\prime \prime}, x^{\prime \prime}, y^{\prime \prime},
$$

with $p_{0}^{\prime \prime}, p_{0,1}^{\prime \prime}, p_{1}^{\prime \prime}, x^{\prime \prime}$ on one line of $E_{2, r}$ and $p_{0}^{\prime \prime}, p_{2}^{\prime \prime}, y^{\prime \prime}$ on another. Since $p_{0}^{\prime \prime}, p_{0,1}^{\prime \prime}, p_{1}^{\prime \prime}, p_{2}^{\prime \prime} \approx p_{0}, p_{0,1}, p_{1}, p_{2} \approx p_{0}^{\prime}, p_{0,1}^{\prime}, p_{1}^{\prime}, p_{2}^{\prime}$ and the two congruent triples $p_{0}^{\prime}, p_{0,1}^{\prime}, p_{1}^{\prime}$ and $p_{0}^{\prime \prime}, p_{0,1}^{\prime \prime}, p_{1}^{\prime \prime}$ both lie on lines, there is a congruent transformation of $E_{2, r}$ on itself mapping $p_{0}^{\prime \prime}, p_{0,1}^{\prime \prime}, p_{1}^{\prime \prime}, p_{2}^{\prime \prime}$ on $p_{0}^{\prime}, p_{0,1}^{\prime}, p_{1}^{\prime}, p_{2}^{\prime}$, respectively. This transformation maps $x^{\prime \prime}$ on that unique point $x^{*}$ of $E_{1, r}\left(p_{0}^{\prime}, p_{0,1}^{\prime}, p_{1}^{\prime}\right)$ with distances from $p_{0}^{\prime}, p_{0,1}^{\prime}, p_{1}^{\prime}$ the same as those of $x^{\prime}$ and so $x^{*}=x^{\prime}$. Similarly, $y^{\prime \prime}$ is sent into that point $y^{*}$ of $E_{1, r}\left(p_{0}^{\prime}, p_{2}^{\prime}\right)$ whose distances from the two non-diametral points $p_{0}^{\prime}, p_{2}^{\prime}$ are the same as those 
of $y^{\prime}$. Thus $y^{*}=y^{\prime}, x y=x^{\prime \prime} y^{\prime \prime}=x^{\prime} y^{\prime}$, and the two line-sums are congruent. That this congruence between the two sets is the only one in which $p_{0}, p_{0,1}, p_{1}, p_{2}$ correspond to $p_{0}^{\prime}, p_{0,1}^{\prime}, p_{1}^{\prime}, p_{2}^{\prime}$, respectively, follows at once since this correspondence determines congruences (a) and (b).

Definition. If $p$ is any point and $\Sigma_{1, r}$ any line of $\Sigma_{r}$ there exists at least one point $f(p)$ of $\Sigma_{1, r}$ (closed and compact) such that $p f(p) \leqq p x, x \in \Sigma_{1, r}$. Call $f(p)$ a foot of $p$ on $\Sigma_{1, r}$, the point $d(f(p))$, diametral to $f(p)$ on $\Sigma_{1, r}(p, f(p))$, a pole of $\Sigma_{1, r}$, and $p f(p)$, the distance of $p$ from $\Sigma_{1, r}$, denoted by dist. $\left(p, \Sigma_{1, r}\right)$.

THEOREM 10. A pole of a line $\Sigma_{1, r}$ has distance $\pi r / 2$ from each point of the line (and hence distance $\pi r / 2$ from the line).

Proof. Let $f(p)$ be a foot of $p$ on $\Sigma_{1, r}, d(f(p))$ a pole of $\Sigma_{1, r}$, and $q$ any point of $\Sigma_{1, r}$. By Corollary 1 , Theorem 8 , the sum of the two intersecting lines $\Sigma_{1, r}, \Sigma_{1, r}(p, f(p), d(f(p)))$ is imbeddable in $E_{\varepsilon, r}$ and hence

$$
\Sigma_{1, r}(q, f(p))+\Sigma_{1, r}(p, f(p), d(f(p))) \approx E_{1, r}\left(q^{\prime}, f\left(p^{\prime}\right)\right)+E_{1, r}\left(p^{\prime}, f\left(p^{\prime}\right), d\left(f\left(p^{\prime}\right)\right)\right),
$$

with $f\left(p^{\prime}\right)$ a foot of $p^{\prime}$ on $E_{1, r}\left(q^{\prime}, f\left(p^{\prime}\right)\right)$ and $d\left(f\left(p^{\prime}\right)\right)$ the point of $E_{1, r}\left(p^{\prime}, f\left(p^{\prime}\right), d\left(f\left(p^{\prime}\right)\right)\right)$ diametral to $f\left(p^{\prime}\right)$. By an elementary property of the elliptic plane, it follows that $q^{\prime} d\left(f\left(p^{\prime}\right)\right)=q d(f(p))=\pi r / 2$.

TheOREM 11. The sums $\Sigma_{1, r}+(p)$ and $E_{1, r}+\left(p^{\prime}\right)$ are congruent if and only if $\operatorname{dist.}\left(p, \Sigma_{1, r}\right)=\operatorname{dist} .\left(p^{\prime}, E_{1, r}\right)$.

Proof. The theorem follows from Theorem 6 in case dist. $\left(p, \Sigma_{1, r}\right)=0$, and from Theorem 10 if the distance is $\pi r / 2$. Suppose, now, $0<$ dist. $\left(p, \Sigma_{1, r}\right)$ $=$ dist. $\left(p^{\prime}, E_{1, r}\right)<\pi r / 2$, let $f(p), f\left(p^{\prime}\right)$ be feet of $p, p^{\prime}$, on $\Sigma_{1, r}, E_{1, r}$, respectively, and $d(f(p)), d\left(f\left(p^{\prime}\right)\right)$ poles on $\Sigma_{1, r}(p, f(p)), E_{1, r}\left(p^{\prime}, f\left(p^{\prime}\right)\right)$, respectively. If $q \in \Sigma_{1, r}(q \neq f(p))$ let $q^{\prime}$ of $E_{1, r}$ correspond to $q$ in any congruence between $\Sigma_{1, r}$ and $E_{1, r}$ which maps $f(p)$ on $f\left(p^{\prime}\right)$.

By Theorem 10, $q d(f(p))=\pi r / 2=q^{\prime} d\left(f\left(p^{\prime}\right)\right)$ and the two quadruples $p, q, f(p), d(f(p))$ and $p^{\prime}, q^{\prime}, f\left(p^{\prime}\right), d\left(f\left(p^{\prime}\right)\right)$ are seen to have all corresponding distances equal except, perhaps, $p q$ and $p^{\prime} q^{\prime}$. But $p, q, f(p), d(f(p))$ $\approx p^{\prime \prime}, q^{\prime \prime}, f\left(p^{\prime \prime}\right), d\left(f\left(p^{\prime \prime}\right)\right)$ of $E_{2, r}$ (Theorem 2) and hence this elliptic quadruple has five of its six distances equal to the corresponding five distances of the elliptic quadruple $p^{\prime}, q^{\prime}, f\left(p^{\prime}\right), d\left(f\left(p^{\prime}\right)\right)$. It follows that the sixth pair of corresponding distances are also equal and the two quadruples are congruent $\left.{ }^{15}\right)$. Hence $p^{\prime} q^{\prime}=p^{\prime \prime} q^{\prime \prime}=p q$ and $\Sigma_{1, r}+(p) \approx E_{1, r}+\left(p^{\prime}\right)$. Since the necessity is obvious, the theorem is established.

5. Linear subspaces of $\Sigma_{r}$. Defining a zero-dimensional subspace $\Sigma_{0, r}$ of $\Sigma_{r}$ as consisting of a single point, it follows from the preceding section that for $k=1$, (i) a $k$-dimensional subspace $\Sigma_{k, r}$ is the locus of all points of $\Sigma_{r}$ on a line with a point of $\Sigma_{k-1, r}$ and a point not belonging to it, (ii) if $p_{0}, p_{1}, \cdots, p_{k}$ are not elements of a $(k-1)$-dimensional subspace $\Sigma_{k-1, r}$ there

(15) They are, in fact, even superposable. 
is one and only one subspace $\Sigma_{k, r}$ of $k$ dimensions containing them, (iii) $\Sigma_{k, r}$ is a linear space, (iv) $\Sigma_{k, r}$ is congruent with $E_{k, r}$, and (v) two distinct (k-1)dimensional subspaces contained in a $\Sigma_{k, r}$ have a $\Sigma_{k-2, r}$ in common( $\left.{ }^{16}\right)$.

If, now, for a given integer $k, \Sigma_{r}$ contains a subspace $\Sigma_{k, r}$ and a point $p$ not belonging to it, a subspace $\Sigma_{k+1, r}$, sometimes denoted by $\left\{p ; \Sigma_{k, r}\right\}$, is defined as the locus of all points of $\Sigma_{r}$ on a line with $p$ and a point of $\Sigma_{k, r}$. We make the inductive hypothesis that all properties of $\Sigma_{1, r}$ proved in the foregoing (in particular, those properties listed above) are valid for every $\Sigma_{k, r}$ $(k=1,2, \cdots, n)$ and shall establish those properties (which are pertinent to our purpose) for $(n+1)$-dimensional subspaces $\Sigma_{n+1, r}$.

Consider an elliptic $(n+1)$-dimensional space $E_{n+1, r}$, an $n$-dimensional subspace $E_{n, r}$ and a point $p^{\prime}$ of $E_{n+1, r}$ with dist. $\left(p^{\prime}, E_{n, r}\right)=$ dist. $\left(p, \Sigma_{n, r}\right)\left({ }^{17}\right)$. Let $f(p), f\left(p^{\prime}\right)$ denote feet of $p, p^{\prime}$ on $\Sigma_{n, r}, E_{n, r}$, respectively, and let $q, q^{\prime}$ be corresponding points in any congruence $\Sigma_{n, r} \approx E_{n, r}$ (inductive hypothesis) which associates $f(p)$ and $f\left(p^{\prime}\right)$. To show that $p q=p^{\prime} q^{\prime}$ suppose $q \neq f(p)$ (trivial, otherwise) and note that $f(p)$ a foot of $p$ on $\Sigma_{n, r}$ implies $f(p)$ a foot of $p$ on $\Sigma_{1, r}(q, f(p))$ which, by the linearity of $\Sigma_{n, r}$ (inductive hypothesis), is contained in $\Sigma_{n, r}$, and hence dist. $\left(p, \Sigma_{1, r}(q, f(p))\right)=\operatorname{dist.}\left(p^{\prime}, E_{1, r}\left(q^{\prime}, f\left(p^{\prime}\right)\right)\right)$. It follows (Theorem 11) that $\Sigma_{1, r}(q, f(p))+(p)$ is congruent to $E_{1, r}\left(q^{\prime}, f\left(\dot{p}^{\prime}\right)\right)$ $+\left(p^{\prime}\right)$ and so $p q=p^{\prime} q^{\prime}$, from which an extension of Theorem 11 is obtained:

THEOREM 12. The sums $\Sigma_{n, r}+(p)$ and $E_{n, r}+\left(p^{\prime}\right)$ are congruent if and only if dist. $\left(p, \Sigma_{n, r}\right)=\operatorname{dist} .\left(p^{\prime}, E_{n, r}\right)$.

ThEOREM 13. Each $(n+1)$-dimensional subspace $\Sigma_{n+1, r}$ of $\Sigma_{r}$ is congruent with the elliptic space $E_{n+1, r}$.

Proof. Let $\Sigma_{n+1, r}=\left\{p_{0} ; \Sigma_{n, r}\right\}$ and consider first the case in which $0<$ dist. $\left(p_{0}, \Sigma_{n, r}\right)<\pi r / 2$. Select in $E_{n+1, r}$ a subspace $E_{n, r}$ and a point $p_{0}^{\prime}$ such that dist. $\left(p_{0}^{\prime}, E_{n, r}\right)=\operatorname{dist} .\left(p_{0}, \Sigma_{n, r}\right)$, and let $f\left(p_{0}\right), f\left(p_{0}^{\prime}\right)$ be feet of $p_{0}$, $p_{0}^{\prime}$ on $\Sigma_{n, r}, E_{n, r}$, respectively.

Consider the congruence $\Sigma_{n, r}+\left(p_{0}\right) \approx E_{n, r}+\left(p_{0}^{\prime}\right)$ (Theorem 12) which associates $f\left(p_{0}\right)$ with $f\left(p_{0}^{\prime}\right)$. If, now, $x \in \Sigma_{n+1, r}$, let $p$ be the point of $\Sigma_{n, r}$ such that $p_{0}, p, x$ are on a line and $p^{\prime}$ the point of $E_{n, r}$ corresponding to $p$ in the above congruence. Since $p_{0} p=p_{0}^{\prime} p^{\prime}$ (Theorem 12), $\Sigma_{1, r}\left(p_{0}, p\right) \approx E_{1, r}\left(p_{0}^{\prime}, p^{\prime}\right)$. Assuming for the present that $p_{0} p \neq \pi r / 2$, let $x^{\prime}$ be the (unique) point of $E_{1, r}\left(p_{0}^{\prime}, p^{\prime}\right)$ corresponding to $x$ in this congruence. We wish to show this mapping (defined for all points $x$ of $\Sigma_{n+1, r}$ such that $p_{0} p \neq \pi r / 2$ ) a congruent one.

Let $x, x^{\prime}$ and $y, y^{\prime}$ be corresponding points in this mapping, with $p_{0}, p, x$ $\approx p_{0}^{\prime}, p^{\prime}, x^{\prime}$ and $p_{0}, q, y \approx p_{0}^{\prime}, q^{\prime}, y^{\prime}$ (all triples lying on lines). If $p=q$ then $x, y \in \Sigma_{1, r}\left(p_{0}, p\right)$ and $x y=x^{\prime} y^{\prime}$ by an above congruence. If $p \neq q$, let $s$ be a

(16) For $k=1, \Sigma_{k-2, r}$ denotes a space whose point set is empty.

(17) The notions dist. $\left(p, \Sigma_{n, r}\right)$, foot of $p$ on $\Sigma_{n, r}$, and so on, are defined as for $\Sigma_{1 . r}$. 
point of $\Sigma_{1, r}(p, q), p \neq s \neq q$, and $s^{\prime}$ the corresponding point of $E_{1, r}\left(p^{\prime}, q^{\prime}\right)\left({ }^{18}\right)$. Noting that the two congruent quadruples $p_{0}, p, q, s$ and $p_{0}^{\prime}, p^{\prime}, q^{\prime}, s^{\prime}$ satisfy the conditions of Theorem 9, we have

$$
\Sigma_{1, r}\left(p_{0}, p\right)+\Sigma_{1, r}(p, q, s) \approx E_{1, r}\left(p_{0}^{\prime}, p^{\prime}\right)+E_{1, r}\left(p^{\prime}, q^{\prime}, s^{\prime}\right)
$$

and hence $q x=q^{\prime} x^{\prime}$. Applying the same theorem to the congruent quadruples $p_{0}, p, x, q$ and $p_{0}^{\prime}, p^{\prime}, x^{\prime}, q^{\prime}$ gives

$$
\Sigma_{1, r}\left(p_{0}, q\right)+\Sigma_{1, r}\left(p_{0}, p, x\right) \approx E_{1, r}\left(p_{0}^{\prime}, q^{\prime}\right)+E_{1, r}\left(p_{0}^{\prime}, p^{\prime}, x^{\prime}\right)
$$

and hence $x y=x^{\prime} y^{\prime}\left({ }^{19}\right)$.

It remains to define the mapping (and prove it a congruence) for points $x$ of $\Sigma_{n+1, r}$ such that $p_{0} p=\pi r / 2$. Now there exists an infinite sequence $\left\{x_{i}\right\}$ of distinct points of $\Sigma_{r}$ with $\lim _{i \rightarrow \infty} x_{i}=x$ and $p_{0} p_{i} \neq \pi r / 2$, where $p_{i} \in E_{n, r}$ with $p_{0}, p_{i}, x_{i}$ on a line $(i=1,2, \cdots)$. For if no such sequence exists then $\Sigma_{n, r}$ contains a neighborhood $N$ of $p$, each point of which is diametral to $p_{0}$. By Theorem 12 each point of $N^{\prime}$, the neighborhood of $p^{\prime}$, which corresponds to $N$ in the congruence $\Sigma_{n, r}+p_{0} \approx E_{n, r}+\left(p_{0}^{\prime}\right)$, has distance $\pi r / 2$ from $p_{0}^{\prime}$ and hence $p_{0}^{\prime}$ is the pole of $E_{n, r}$; that is, $p_{0}^{\prime} x^{\prime}=\pi r / 2$ for every point $x^{\prime}$ of $E_{n, r}$. This is impossible since $p_{0}^{\prime} f\left(p_{0}^{\prime}\right)=p_{0} f\left(p_{0}\right)=$ dist. $\left(p_{0}, \Sigma_{n, r}\right)<\pi r / 2$.

Let $x_{i}^{\prime}$ be the unique point of $\Sigma_{n+1, r}$ corresponding to $x_{i}$ by means of the mapping previously defined. Since $\left\{x_{i}\right\}$ has limit $x$ it is a Cauchy sequence and since (by the first part of the proof) $x_{i} x_{j}=x_{i}^{\prime} x_{j}^{\prime}(i, j=1,2, \cdots)$, sequence $\left\{x_{i}^{\prime}\right\}$ is also a Cauchy sequence. The elliptic space $E_{n, r}$ being complete contains a unique point $x^{\prime}=\lim _{i \rightarrow \infty} x_{i}^{\prime}$. If we let $x$ and $x^{\prime}$ correspond, the continuity of the metric insures $x y=x^{\prime} y^{\prime}$. Thus the existence of a congruent mapping of $\Sigma_{n+1, r}$ onto $E_{n+1, r}$ is established, and the theorem is proved in this case $\left({ }^{20}\right)$.

It remains to consider the case in which $\Sigma_{n+1, r}=\left\{p_{0} ; \Sigma_{n, r}\right\}$, dist. $\left(p_{0}, \Sigma_{n, r}\right)$ $=\pi r / 2$. Let $q_{0}$ be a point of $\Sigma_{1, r}\left(p_{0}, f\left(p_{0}\right)\right), q_{0} \neq p_{0}, f\left(p_{0}\right)$, and $\Sigma_{n+1, r}^{*}$ $=\left\{q_{0} ; \Sigma_{n, r}\right\}$. Then $f\left(p_{0}\right)$ is a foot of $q_{0}$ on $\Sigma_{n, r}$ (for if $p \in \Sigma_{n_{r} r}$, then $\left.q_{0} p \geqq p_{0} p-p_{0} q_{0} \geqq p_{0} f\left(p_{0}\right)-p_{0} q_{0}=q_{0} f\left(p_{0}\right)\right)$ and since $0<q_{0} f\left(p_{0}\right) \neq \pi r / 2$, then $0<$ dist. $\left(q_{0}, \Sigma_{n, r}\right)<\pi r / 2$. It follows from the above that $\Sigma_{n+1, r}^{*} \approx E_{n+1, r}$. If, now, $x \in \Sigma_{n+1, r}=\left\{p_{0} ; \Sigma_{n, r}\right\}$ and $p \in \Sigma_{n, r}$ with $p_{0}, p, x$ on a line, the above congruence maps these three points onto points $p_{0}^{\prime}, p^{\prime}, x^{\prime}$ (since it maps $\Sigma_{1, r}\left(p_{0}, p\right)$ onto $\left.E_{1, r}\left(p_{0}^{\prime}, p^{\prime}\right)\right)\left({ }^{21}\right)$. Since $E_{1, r}\left(q_{0}^{\prime}, x^{\prime}\right)$ intersects $E_{n, r}, \Sigma_{1, r}\left(q_{0}, x\right)$ meets $\Sigma_{n, r}$ and so $x \in\left\{q_{0} ; \Sigma_{n, r}\right\}$. In this way it is seen that $\Sigma_{n+1, r}=\Sigma_{n+1, r}^{*}$ $\approx E_{n+1, r}$ and the proof of the theorem is complete.

COROLLARY. The $(n+1)$-dimensional subspace $\Sigma_{n+1, r}$ is a linear space.

(18) Since $\Sigma_{n, r}$ is a linear space, $s \in \Sigma_{1, r}(p, q), p, q \in \Sigma_{n, r}$ imply $s \in \Sigma_{n, r}$.

(19) In case $p=x$ or $q=y$ (disjunctively) only one application of Theorem 9 is needed, while if $p=x$ and $q=y, x y=x^{\prime} y^{\prime}$ from the congruence of the two $n$-spaces.

(20) That the mapping is onto is clear since $E_{n+1, r}=\left\{p_{0}^{\prime} ; E_{n, r}\right\}$.

(21) Since $p_{0}, q_{0}, f\left(p_{0}\right)$ are on a line $p_{0} \in\left\{q_{0} ; \Sigma_{n, r}\right\}$. 
Theorem 14. Any $n+2$ points $p_{0}, p_{1}, \cdots, p_{n+1}$ of $\Sigma_{r}$ which are not contained in any $n$-dimensional subspace lie in one and only one $(n+1)$-dimensional subspace.

Proof. The points $p_{1}, p_{2}, \cdots, p_{n+1}$ are not in any $\Sigma_{n-1, r}$ (for otherwise, since then $p_{0} \bar{\in} \Sigma_{n-1, r}$, this point and subspace generate a subspace $\Sigma_{n, r}$ which contains $p_{0}, p_{1}, \cdots, p_{n+1}$, contrary to hypothesis) and hence (inductive hypothesis) are in one and only one $\Sigma_{n, r}$. Then $p_{0}$ and $\Sigma_{n, r}$ generate a subspace $\Sigma_{n+1, r}$ containing $p_{0}, p_{1}, \cdots, p_{n+1}$. If, now, $\Sigma_{n+1, r}^{*}$ also contains these $n+1$ points then (from the linearity of all subspaces) it contains the unique $\Sigma_{n, r}$ determined by $p_{1}, p_{2}, \cdots, p_{n+1}$ and it follows that $\Sigma_{n+1, r}^{*} \supset \Sigma_{n+1, r}$. On the other hand, it is easy to show that the point and $n$-dimensional subspace generating $\Sigma_{n+1, r}^{*}$ are contained in $\Sigma_{n+1, r}$ and so $\Sigma_{n+1, r} \supset \Sigma_{n+1, r}^{*}\left({ }^{22}\right)$.

6. The characterization theorems. We have seen that for every positive integer $n$, each set of $n+1$ points of $\Sigma_{r}$ either lies in an $(n-1)$-dimensional linear subspace or determines uniquely an $n$-dimensional linear subspace, and all linear subspaces of $\Sigma_{r}$ are congruent with elliptic spaces of corresponding dimensions and space constant $r$. The following theorem results:

Characterization Theorem I. The space $\Sigma_{r}$ is congruent with an elliptic space of finite or infinite dimension and space constant $r$.

An additional postulate is needed for the congruence of $\Sigma_{r}$ with an elliptic space of given finite dimension. We introduce two postulates (one global and the other a localization of it) the adjunction of either of which to the set of postulates defining $\Sigma_{r}$ yields a space congruent with the elliptic space $E_{n, r}$.

Postulate VII (GLOBAL). There is a positive integer $k$ such that each set of $k+2$ points $p_{0}, p_{1}, \cdots, p_{k+1}$ of $\Sigma_{r}$ has the property that if an $\epsilon$-matrix $\left(\epsilon_{i j}\right)$ exists for which no principal minor of $\left|\epsilon_{i j} \cos \left(p_{i} p_{i} / r\right)\right|(i, j=0,1, \cdots, k+1)$ is negative, then there is at least one $\epsilon$-matrix for which the determinant vanishes and has no principal minor negative.

Denoting by Postulate VII $_{n}$ (Global) the above postulate in case $n$ is the smallest member of the class $\{k\}$ of integers described in it, we prove:

Characterization Theorem II. A necessary and sufficient condition that $\Sigma_{r}$ be congruent with the n-dimensional elliptic space $E_{n, r}$ is that it satisfy Postulate $\mathrm{VII}_{n}$ (Global).

Proof. The necessity follows at once from Result 1.

To prove the sufficiency, note first that $\Sigma_{r}$ contains $n+1$ points which are

(22) It follows from the congruence of $\Sigma_{n+1, r}$ with $E_{n+1, r}$ that any line of $\Sigma_{n+1, r}$ intersects any $n$-dimensional subspace $\Sigma_{n, r}$. Hence $\Sigma_{1, r}\left(p_{0}, p\right)$ intersects $\Sigma_{n_{1}, r} p \in \Sigma_{n, r}^{*}+\left(p_{0}^{*}\right), \Sigma_{n_{+1, r}^{*}}$ $=\left\{p_{0}^{*} ; \Sigma_{n, r}^{*}\right\}$, and so $\Sigma_{n, r}^{*}+\left(p_{0}^{*}\right)$ is a subset of $\Sigma_{n+1, r}$.

Another argument is that $\Sigma_{n+1, r}^{*} \approx E_{n+1, r} \approx \Sigma_{n+1, r}$ and $\Sigma_{n+1, r}^{*} \supset \Sigma_{n+1, r}$ imply that the two $(n+1)$-dimensional subspaces are identical, since both are compact. 
not imbeddable in $E_{n-1, r} ;$ for in the contrary case it follows by Result 1 that Postulate VII $_{n-1}$ (Global) holds in $\Sigma_{r}$, contrary to hypothesis. Since $\Sigma_{n-1, r}$ $\approx E_{n-1, r}$, a set of $n+1$ points not imbeddable in $E_{n-1, r}$ is not congruently contained (and hence not contained) in any $\Sigma_{n-1, r}$ and therefore determines uniquely an $n$-dimensional subspace $\Sigma_{n, r}$ of $\Sigma_{r}$ (Theorem 14) which is congruent with $E_{n, r}$.

To complete the proof we show that each point of $\Sigma_{r}$ belongs to $\Sigma_{n, r}$. In the contrary case, suppose $p \in \Sigma_{r}$ and $p \bar{E} \Sigma_{n, r}$. Then $p$ and $\Sigma_{n, r}$ generate a unique $(n+1)$-dimensional subspace $\Sigma_{n+1, r}$ of $\Sigma_{r}$ which is congruent to $E_{n+1, r}$, and therefore contains $n+2$ points $p_{0}, p_{1}, \cdots, p_{n+1}$ with $p_{i} p_{j}=\pi r / 2$ $(i, j=0,1, \cdots, n+1 ; i \neq j)$. Now the $\epsilon$-matrix $\left(\epsilon_{i j}\right), \epsilon_{i j}=\epsilon_{j i}=1(i, j=0,1$, $\cdots, n+1)$, is such that $\left|\epsilon_{i j} \cos \left(p_{i} p_{j} / r\right)\right|(i, j=0,1, \cdots, n+1)$ has no principal minor negative, but obviously no $\epsilon$-matrix exists for which the determinant vanishes. Thus $\Sigma_{r}=\Sigma_{n, r} \approx E_{n, r}$ and the theorem is proved.

A given space has congruence order $m$ with respect to a given class of spaces provided each member of the class is congruently contained in the given space whenever each $m$ of its points has that property. It has been shown that for every positive integer $k$, each $\Sigma_{k, r}$ of $\Sigma_{r}$ is congruent with $E_{k, r}$, and so for every positive integer $k$, each $k+1$ points of $\Sigma_{r}$ are imbeddable in $E_{k, r .}$. Hence by Result 1 , for every positive integer $k$ there corresponds to each set of $k+1$ points $p_{0}, p_{1}, \cdots, p_{k}$ of $\Sigma_{r}$ an $\epsilon$-matrix $\left(\epsilon_{i j}\right)$ such that $\left|\epsilon_{i j} \cos \left(p_{i} p_{j} / r\right)\right|(i, j=0,1, \cdots, k)$ has none of its principal minors negative. Then Postulate VII $_{n}$ (Global) and Result 1 imply that every $n+2$ points of $\Sigma_{r}$ are imbeddable in $E_{n, r}$.

COROLlaRY. The n-dimensional elliptic space $E_{n, r}$ has congruence order $n+2$ with respect to the class of spaces $\Sigma_{r}\left({ }^{23}\right)$.

Localizing Postulate $\mathrm{VII}_{n}$ (Global), suppose there is a point $q_{0}$ of $\Sigma_{r}$ and a spherical neighborhood $S\left(q_{0} ; \pi r / 6\right)$ of it (the set of all points $x$ of $\Sigma_{r}$ with $q_{0} x<\pi r / 6$ ) in which it is satisfied. Then (as in the proof of the preceding theorem) $S\left(q_{0} ; \pi r / 6\right)$ contains $n+1$ points $p_{0}, p_{1}, \cdots, p_{n}$ which are not congruently imbeddable in $E_{n-1, r}$ and so are not contained (congruently or actually) in any $\Sigma_{n-1, r .}$. These points determine a unique $n$-dimensional subspace $\Sigma_{n, r}\left(p_{0}, p_{1}, \cdots, p_{n}\right)$ which we show contains $S\left(q_{0} ; \pi r / 6\right)$.

Let $x$ be any element of $S\left(q_{0} ; \pi r / 6\right)$. If $p_{0}, p_{1}, \cdots, p_{n}, x$ are in any $\Sigma_{n, r}$ they are in $\Sigma_{n, r}\left(p_{0}, p_{1}, \cdots, p_{n}\right)$. If they do not belong to any $n$-dimensional subspace they determine a unique $\Sigma_{n+1, r}\left(p_{0}, p_{1}, \cdots, p_{n}, x\right)$. Denoting by $p_{0}^{\prime}, p_{1}^{\prime}, \cdots, p_{n}^{\prime}, x^{\prime}$ the points $E_{n+1, r}$ corresponding to $p_{0}, p_{1}, \cdots, p_{n}, x$

(23) What congruence order (if any) $E_{n, r}$ has with respect to the class $\{S\}$ of all semimetric spaces is an unsolved problem. The elliptic line has congruence order 4 with respect to $\{S\}$ but, as noted in (e), $\$ 2$, the least possible congruence order of $E_{2, r}$ is 7 . Since $E_{n, r}$ is compact metric, it has hyperfinite congruence order with respect to all separable semimetric spaces; that is, each such space is congruently contained in $E_{n, r}$ whenever all of its finite subsets are. 
in a congruence $\Sigma_{n+1, r} \approx E_{n+1, r}$, it is clear that the "primed" points are not in any $E_{n, r}\left({ }^{24}\right)$. On the other hand, $p_{0}, p_{1}, \cdots, p_{n}, x \in S\left(q_{0} ; \pi r / 6\right)$, implies (by

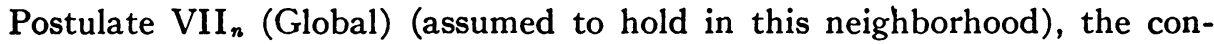
gruence of all linear subspaces with elliptic spaces, and Result 1) the existence of points $p_{0}^{\prime \prime}, p_{1}^{\prime \prime}, \cdots, p_{n}^{\prime \prime}, x^{\prime \prime}$ of an $E_{n, r}$ congruent to them. Thus $p_{0}^{\prime}, p_{1}^{\prime}, \cdots, p_{n}^{\prime}, x^{\prime} \approx p_{0}^{\prime \prime}, p_{1}^{\prime \prime}, \cdots, p_{n}^{\prime \prime}, x^{\prime \prime}$ with the "primed" points in $E_{n+1, r}$ and no lower-dimensional elliptic space, and the "double primed" points in $E_{n, r}\left({ }^{25}\right)$.

It follows that $\epsilon$-matrices $\left(\epsilon_{i j}\right),\left(\epsilon_{i j}^{\prime}\right)$ exist such that (i) $\left|\epsilon_{i j} \cos \left(p_{i} p_{j} / r\right)\right|$ $\left(i, j=0,1, \cdots, n+1 ; p_{n+1}=x\right)$ vanishes, (ii) $\left|\epsilon_{j}^{\prime} \cos \left(p_{i} p_{j} / r\right)\right|(i, j=0,1$, $\left.\cdots, n+1 ; p_{n+1}=x\right)$ does not vanish, and in each determinant all nonvanishing principal minors are positive. Making the (obviously permissible) selection $\epsilon_{0 j}=\epsilon_{j 0}=\epsilon_{0 j}^{\prime}=\epsilon_{j 0}^{\prime}=1 \quad(j=0,1, \cdots, n+1)$ and noting that, since $p_{0}, p_{1}, \cdots, p_{n}, x$ are points of $S\left(q_{0} ; \pi r / 6\right), \Delta^{*}<0$ for each three of these points, it follows at once that every $\epsilon_{i j}$ and $\epsilon_{y j}^{\prime}$ equals $1(i, j=0,1, \cdots, n+1)$, and the properties (i) and (ii) are contradictory. Thus $p_{0}, p_{1}, \cdots, p_{n}, x$ are elements of an $n$-dimensional subspace and so $S\left(q_{0} ; \pi r / 6\right)$ is a subset of $\Sigma_{n, r}\left(p_{0}, p_{1}, \cdots, p_{n}\right)$.

If, now, $x \in \Sigma_{r}, x \neq q_{0}$, then $x \in \Sigma_{1, r}\left(q_{0}, x\right)$. Since this line clearly has two distinct points in common with $S\left(q_{0} ; \pi r / 6\right)$, it has two distinct points in common with the linear subspace $\Sigma_{n, r}\left(p_{0}, p_{1}, \cdots, p_{n}\right)$ and therefore is contained in this subspace. Thus $\Sigma_{n, r}\left(p_{0}, p_{1}, \cdots, p_{n}\right)$ contains every point of $\Sigma_{r}$, which gives $\Sigma_{r}=\Sigma_{n, r} \approx E_{n, r}$. These considerations lead to the following postulate and theorem :

Postulate VII (LOCAL). There exists an integer $k$, a point $q_{0}$ of $\Sigma_{r}$ and a spherical neighborhood $S\left(q_{0} ; \pi r / 6\right)$ of $q_{0}$ such that each set of $k+2$ points $p_{0}, p_{1}, \cdots, p_{k+1}$ of $S\left(q_{0} ; \pi r / 6\right)$ has the property that if an $\epsilon$-matrix $\left(\epsilon_{i j}\right)$ exists for which no principal minor of the determinant $\left|\epsilon_{i j} \cos \left(p_{i} p_{j} / r\right)\right|(i, j=0,1$, $\cdots, k+1)$ is negative, then there is at least one $\epsilon$-matrix for which no principal minor of the corresponding determinant is negative and the determinant vanishes.

Characterization Theorem III. A necessary and sufficient condition that $\Sigma_{r}$ be congruent with the n-dimensional elliptic space $E_{n, r}$ is that it satisfy Postulate $\mathrm{VII}_{n}$ (Local).

Suppose now that Postulate VII (Global) is not valid in $\Sigma_{r}$. Then for every positive integer $k$, (1) every set of $k+1$ points of $\Sigma_{r}$ is congruently imbeddable in $E_{k, r}$ (since $\Sigma_{k, r} \approx E_{k, r}$ ) and (2) $\Sigma_{r}$ contains a set of $k+2$ points

${ }^{(24)}$ If $p_{0}^{\prime}, p_{1}^{\prime}, \cdots, p_{n}^{\prime}, x^{\prime} \in E_{n, r}$ then (since the points $p_{0}, p_{1}, \cdots, p_{n}$ are not congruently imbeddable in any $\left.E_{n-1, r}\right) p_{0}^{\prime}, p_{1}^{\prime}, \cdots, p_{n}^{\prime}$ do not lie in any $E_{n-1, r}$ and so $x^{\prime} \in E_{n, r}\left(p_{0}^{\prime}, p_{1}^{\prime}, \cdots, p_{n}^{\prime}\right)$; that is, $x^{\prime}$ is on a line with $p_{0}^{\prime}$ and a point of $E_{n-1, r}\left(p_{1}^{\prime}, p_{2}^{\prime}, \cdots, p_{n}^{\prime}\right)$. Then $\Sigma_{n+1, r} \approx E_{n+1, r}$ implies $x$ is on a line with $p_{0}$ and a point of $\Sigma_{n-1, r}\left(p_{1}, p_{2}, \cdots, p_{n}\right)$; that is, $x \in \Sigma_{n, r}\left(p_{0}, p_{1}, \cdots, p_{n}\right)$.

${ }^{(25)}$ From $\S 2,(a),(b),(c)$, this is not a priori impossible. 
not congruently contained in $E_{k, r}$. It follows from Characterization Theorem III that property (2) belongs also to the neighborhood $S(p ; \pi r / 6)$ of every point $p$ of $\Sigma_{r}$, while $S(p ; \pi r / 6)$ has, of course, property (1) as a subset of $\Sigma_{r}$.

That part of the argument preceding the third characterization theorem which refers to the determinant $\left|\epsilon_{i j} \cos \left(p_{i} p_{j} / r\right)\right|$ formed for points of $S\left(q_{0}\right.$; $\pi r / 6)$ permits now the statement that (1) for every positive integer $k$ and every $k+1$ points $p_{0}, p_{1}, \cdots, p_{k}$ of $S(p ; \pi r / 6)$, the determinant $\left|\cos \left(p_{i} p_{j} / r\right)\right|$ $(i, j=0,1, \cdots, k)$ is non-negative, along with all of its principal minors, and (2) for every positive integer $k, S(p ; \pi r / 6)$ contains $k+1$ points with nonvanishing determinant.

If $S(p ; \pi r / 6)$ is separable its congruence with a subset of the geodesically metrized "surface" of the sphere of radius $r$ in Hilbert space follows, and hence (since $x, y \in S(p ; \pi r / 6)$ implies $x y<\pi r / 3) S(p ; \pi r / 6)$ is congruent with a subset of that space $E_{\infty, r}$ obtained by identifying diametral point-pairs of the surface of the Hilbert sphere of radius $r$. As each point of $\Sigma_{r}$ is on a line with two points of $S(p ; \pi r / 6), \Sigma_{r}$ is congruently contained in $E_{\infty, r}$. The congruence of the two spaces follows easily from the presence in $\Sigma_{r}$ of $k$-dimensional subspaces $\Sigma_{k, r}$ for every integer $k=1,2, \cdots$, and we have:

Characterization Theorem IV. A necessary and sufficient condition that $\Sigma_{r}$ be congruent with the infinitely-dimensional elliptic space $E_{\infty, r}$ is that (i) a spherical neighborhood $S(p ; \pi r / 6)$ be separable and (ii) for each positive integer $k$, $\Sigma_{r}$ contain $k+1$ points $p_{0}, p_{1}, \cdots, p_{k}$ such that for no $\epsilon$-matrix $\left(\epsilon_{i j}\right)$ does the determinant $\left|\epsilon_{i j} \cos \left(p_{i} p_{j} / r\right)\right|(i, j=0,1, \cdots, k)$ vanish and have all of $i t s$ principal minors non-negative.

7. Concluding remarks. It is observed that the local-global weak fivepoint condition expressed by Postulate III might, perhaps, be replaced by a global weak four-point property such as, for example, the one proved in Theorem 2. This property is indeed sufficient to derive all the results of $\$ 3$ up to Theorem 8 , in place of which it may be shown that the sum of any two mutually perpendicular (intersecting) lines of $\Sigma_{r}$ is congruently imbeddable in $E_{2, r .}$ There are difficulties, however, in applying this result to obtain a proof of the principal Theorem 13. The point does not seem important, since any success in weakening slightly Postulate III is likely to be at the cost of somewhat more involved proofs.

We note, finally, certain theorems on elliptic space proved by Busemann in his recent Study of metric methods in Finsler spaces (Annals of Mathematics Studies, no. 8, 1942). On the basis of a set of five axioms which insure metricity, finite-dimensionality, and unique geodesics through every pair of distinct points, so-called S.L. (straight line) spaces are developed, and it is shown that any S.L. space is congruent with a euclidean, elliptic or hyperbolic space whenever each two congruent triples of sufficiently small diameter are superposable. This interesting theorem is not, of course, a metric character- 
ization of the spaces concerned in the sense of the definition given in $\$ 1$. One inquires, "What distance relations must the metric of an S.L. space satisfy in order that superposability of triples may exist in the small?"

Another theorem proves that an S.L. space of dimension greater than 2, in which the spheres are convex, is elliptic if at least one geodesic is closed. Here "convexity" is not metric convexity and the notion "convexity of spheres" is not formulated in terms of distance relations. More important, however, is the failure of the theorem to cover the case of the elliptic plane. Whether or not the theorem is valid for dimension 2 is an unsolved problem.

These theorems are clearly of a different kind than those obtained in this paper. No less different are the character of the proofs and the nature of the basic assumptions.

\section{BIBLIOGRAPHY}

1. L. M. Blumenthal, Concerning spherical spaces, Amer. J. Math. vol. 57 (1935) pp. 51-61.

2. pp. 3-20.

3. - Distance geometries, University of Missouri Studies vol. 13 (1938).

4. - Some imbedding theorems and characterization problems of distance geometry, Bull. Amer. Math. Soc. vol. 49 (1943) pp. 321-338.

5. Karl Menger, Untersuchungen ïber allgemeine Metrik, Math. Ann. vol. 100 (1928) pp. 75-163.

6. W. A. Wilson, $A$ relation between metric and euclidean spaces, Amer. J. Math. vol. 54 (1932) pp. 505-517.

UNIVERSITY OF MISSOURI, Columbia, Mo. 